\title{
Pressure Controlled Ventilation versus Volume-Controlled Ventilation Regarding Hemodynamics and Stress Response for Posterior Lumbar Spine Surgeries in The Prone Position Ahmed Mohamed Reda Ragheb*1, Ahmed ELSaied Abdul Rahman², Ayman Mohamady Aldemrdash ${ }^{1}$, Sherif Kamal Hafez Arafa ${ }^{1}$
}

${ }^{1}$ Department of Anaesthesiology and Intensive care, Faculty of Medicine - Aswan University, Aswan, ${ }^{2}$ Department of Anesthesiology and Intensive care, Faculty of Medicine - Sohag University, Sohag, Egypt

*Corresponding author: Ahmed Mohamed Reda Ragheb,

Mobile: (+20)01006228286, E-Mail: dr_ahmedmorsi@yahoo.com

\begin{abstract}
Background: Prone positioning may induce alterations of airway pressures and hemodynamic that may affect intraoperative blood loss. Ventilation mode may modify these alterations.

Objective: Our study aimed to differences between pressure-controlled ventilation (PCV) and volume-controlled ventilation (VCV) in their effects on hemodynamic changes during posterior lumbar surgeries performed in the prone position.

Patients and Methods: This is a prospective randomized controlled study, which was performed in the Department of Anaesthesiology and Surgical ICU, Aswan university, Egypt at the duration from 1-6-2017 to 13-12-2018 for 60 patients who had posterior lumbar spine surgery in prone position who were eligible to inclusion/exclusion criteria divided into two groups; Group (I) were received VCV mode $(\mathrm{n}=30)$ and Group (II) were received PCV mode $(\mathrm{n}=$ 30) then study parameters were recorded intraoperatively and postoperatively in the two groups of ventilation modes. Results: As regarding heart rate, mean arterial blood pressure, serum glucose level, and serum cortisol level, they were statistically significantly lower in PCV group than VCV group both intraoperative and postoperative which reflects that stress response is lower with PCV more than VCV and that may have also a rule in decreasing intraoperative blood loss and improving the surgical field in these surgeries.

Conclusions: for lumbar spine surgeries in the prone position, PCV was associated with decreased HR, blood pressure, cortisol, glucose levels compared with VCV. The stress response is lesser with PCV which is better for this type of patients.
\end{abstract}

Keywords: Pressure-controlled ventilation, Volume-controlled ventilation, Hemodynamic changes, Lumbar spine surgery.

\section{INTRODUCTION}

In lumbar spine surgery of the posterior approach, the prone position is a common position to be used. It is one of the excellent positions for exposing the back of the patient and gives a clear angle view for vertebral column spines especially lumbar spines ${ }^{(\mathbf{1})}$. Changing the patient's position from supine to prone during general anaesthesia for this kind of surgery has complications on venous pressure of the epidural veins and mechanics of respiration which is reflected in intraoperative stress response and hemodynamic changes ${ }^{(2)}$.

The lumbar spine surgical procedures are widely increasing nowadays especially after rapidly developing surgical techniques in this field and the advancement in medical engineering concerning its equipment. This type of surgery acts as a wide section through hospital admitted patients and most of these patients are presenting with acute surgical indications as in cases of trauma and acute pain of lumbar disc prolapse ${ }^{(3)}$.

During anaesthesia of these surgeries, perioperative complications are common. Among complications, those which are affecting the hemodynamic which may induce high morbidity rates among these patients ${ }^{(4)}$. The position which is applied during surgery and ventilation mode parameters that are selected during general anaesthesia here are affecting the pulmonary mechanics, hemodynamic changes, and stress response. Hemodynamic stability is important for both intraoperative and postoperative stages. The stress response is one of the factors which is reflecting on the hemodynamic stability of the patient. Among parameters of the stress response are heart rate, mean arterial blood pressure, serum glucose level, serum cortisol level, and PH in arterial blood gases ${ }^{(5)}$.

From the above, the prone position is one of the difficult managed and challenging surgical positions with a high incidence of complications ${ }^{(6)}$. Prone positioning may induce alterations of hemodynamic that may affect the outcome ${ }^{(7)}$. Thus, the current study was conducted to test the effects of ventilation modes pressure-controlled ventilation versus volumecontrolled ventilation on hemodynamics during anaesthesia of this type of patients, and if the type of ventilation may modify these alterations.

\section{AIM OF THE WORK}

Clarification of the relation between ventilation mode and stress response and its resulted in 
hemodynamic changes during posterior lumbar spine surgeries performed in the prone position. we compared PCV and VCV modes and our indicators in this study were heart rate (HR), mean arterial blood pressure (MAP), serum glucose level (s. glucose), and serum cortisol level (s. cortisol).

\section{PATIENTS AND METHODS}

Study here is a prospective randomized controlled study was performed in the duration from 1-6-2017 to 13-12-2018 at the Department of Anaesthiology and Surgical ICU, Aswan University, Egypt. The study was done on 60 patients all of them underwent posterior lumbar spine surgery in prone position and were eligible to study's inclusion and exclusion criteria. After the approval was obtained, Preliminary information about the study was provided to the candidate participants who were then provided with detailed information through an informed voluntary consent form and signed the form. Patients were randomly selected by opening sealed envelopes, to receive one of two ventilation modes during general anaesthesia for their surgery, either PCV or VCV. Computerized randomization was done which divided patients of the study into two groups, 30 patients in each group; Group (I): were received VCV mode $(\mathrm{n}=$ 30). Group (II): were received PCV mode $(n=30)$.

Inclusion criteria: Age 20:55 years old. Normal body mass index (BMI 18.5: $29.9 \mathrm{Kg} / \mathrm{m}^{2}$ ). Expected surgery time is not more than 2 hours. No expected massive intra-operative bleeding. Non-smoker. ASA I \& II. No cardiac, respiratory, cerebrovascular, neuromuscular, hepatic, renal nor endocrine diseases. No history of previous lung surgery.

Exclusion criteria: Age $<20$ years or $>55$ years old. Body mass index higher than normal (BMI equal or more than $30 \mathrm{~kg} / \mathrm{m} 2$ ). Expected surgery time more than 2 hours. Expected massive intra-operative bleeding. Smoker. ASA III \& IV. Cardiac, respiratory, cerebrovascular, neuromuscular, hepatic, renal nor endocrine diseases. Previous lung surgery.

The following investigations were checked 24 hours preoperatively to evaluate the basic levels of the patient to compare between these preoperative basic levels with intraoperative and postoperative results in the two groups of ventilation modes of the study: Heart rate. Blood pressure. Serum cortisol level. Glucose level \& Arterial blood gases (ABG).

Basic preoperative laboratory as $\mathrm{CBC}$, coagulation profile, renal function test, liver function test and serum electrolytes were done and radiological investigations as chest $\mathrm{x}$-ray and abdominal ultrasonography were done also for all patients to confirm fulfilling both inclusion and exclusion criteria.

All the patients were planned to fast 7 hours preoperatively, a peripheral intravenous line 20 gauge was secured in the patient's room, premedication of intravenous ranitidine $50 \mathrm{mg}$ and odansetron $8 \mathrm{mg}$ were given one hour before surgery time.

Standard monitors were applied for the patient in the operating room and before starting anaesthesia consisting of non-invasive blood pressure (which was adjusted to measure systolic, diastolic and mean blood pressure every 5 minutes), ECG, pulse oximetry.

Arterial cannula was inserted in the radial artery after Allen's test was satisfactory and after induction of general anaesthesia for extraction of arterial blood gases samples. The monitor and anaesthetic machine which were used in the study were the same type in all patients. The monitor was equipped by $\mathrm{CO} 2$ analyser (capnography) and inhalational anaesthetic gas analyser.

Pre-oxygenation of the patient for 5 minutes with O2 100\% followed by induction of general anaesthesia for all patients in supine position by IV injection of fentanyl $1 \mathrm{mic} / \mathrm{kg}$, Lidocaine (2\%) $0.02 \mathrm{mg} / \mathrm{kg}$ before propofol injection, Propofol $2.5 \mathrm{mg} / \mathrm{kg}$ and muscle relaxation by atracurium $0.5 \mathrm{mg} / \mathrm{kg}$.

Maintenance of general anaesthesia by isoflurane with end tidal concentration of 0.7: 1.1. Atracurium maintenance dose of $0.1 \mathrm{mg} / \mathrm{kg}$ IV was given every 20 minutes starting from induction time. IV fluid by crystalloids was given according to weight, fasting time and intraoperative fluid loss. IV ketorolac $1 \mathrm{mg} / \mathrm{kg}$ with $100 \mathrm{ml}$ normal saline infusion over 30 minutes was given. Paracetamol $1 \mathrm{gm} \mathrm{IV} \mathrm{infusion} \mathrm{was} \mathrm{given} \mathrm{as}$ analgesia for all patients.

Volume controlled ventilation (VCV) group settings were adjusted as $\mathrm{FiO} 2 \quad 50 \%$ (O2:air concentration $=1: 1)$, tidal volume $\left(\mathrm{V}_{\mathrm{T}}\right) 8 \mathrm{ml} / \mathrm{kg}$, RR to maintain an end tidal $\mathrm{CO} 2(\mathrm{ETCO} 2)$ of $33: 35 \mathrm{mmHg}$, positive end expiratory pressure (PEEP) of $0 \mathrm{~cm} \mathrm{H} 2 \mathrm{O}$.

Pressure controlled ventilation (PCV) group settings were adjusted as $\mathrm{FiO} 2 \quad 50 \% \quad(\mathrm{O} 2$ : air concentration $=1: 1$ ). Inspiratory pressure was setted in two times; just after induction of anaesthesia in supine position and once the patient in prone position, at both a targetted tidal volume $\left(\mathrm{V}_{\mathrm{T}}\right)$ of $8 \mathrm{ml} / \mathrm{kg}$ was aimed, $\mathrm{RR}$ was adjusted to maintain an end tidal $\mathrm{CO} 2$ (ETCO2) of $33: 35 \mathrm{mmHg}$, positive end expiratory pressure (PEEP) of $0 \mathrm{~cm} \mathrm{H} 2 \mathrm{O}$.

At the end of the surgery recovery from anaesthesia was done by cessation of isoflurane with IV neostigmine $0.04 \mathrm{mg} / \mathrm{kg}$ and atropine $0.02 \mathrm{mg} / \mathrm{kg}$ after patient started to retain motor power.

After induction of anaesthesia and in supine position, the following parameters were recorded: End tidal Carbon dioxide (ETCO2). Tidal volume $\left(\mathrm{V}_{\mathrm{T}}\right)$. \& Respiratory rate (RR).

30 minutes after prone position, these parameters were recorded: Heart rate. Blood pressure. Serum cortisol level. Glucose level. ETCO2. V $\mathrm{T}$. RR. \& Arterial blood gases for documentation of $\mathrm{PH}, \mathrm{PaO} 2$ and $\mathrm{PaCO} 2$.

One hour postoperatively in the recovery room, these parameters were recorded: Heart rate. Blood pressure. Serum cortisol level. Glucose level. \& Arterial blood gases for documentation of $\mathrm{PH}, \mathrm{PaO} 2$ and $\mathrm{PaCO} 2$. 


\section{Ethical considerations:}

The confidentiality of all participants' data participated in this study was protected and secured. Participating patients were not be identified by name in any publication nor report resulted from collected data in this study. Ethical aspects were implicated in all stages of this study. The purpose and nature of the study as well as the risks were explained to the patients and were clearly understood by them before participants started this study. Patients had the right to terminate participation in this study without affecting the patient's rights in having proper health care in the study site. Patients had rights of questions regarding the study and they are freely given informed consent to participate in this study. They signed an informed consent form. The study was approved by the Ethics Board of Aswan University.

\section{Statistical methods}

Analysis of the resulted data was described using the mean and standard deviations (SD). The results of the evaluation were considered to be statistically significant when p-values were less than 0.05 . The compliances of the variables with the normal distribution were analyzed. Wilcoxon signed ranks test was used for comparison between dependent variables, while the Mann-Whitney $\mathrm{U}$ test was used for comparison of independent variables. The Student t-test was used for independent continuous variables with the normal distribution. A paired-samples t-test was used for the comparison of dependent variables with the normal distribution.

\section{RESULTS}

Comparison between the two groups of ventilation group (I) and group (II) was done in these points: Heart rate, mean arterial pressure, serum cortisol level, and serum glucose level (preoperative, intraoperative in the prone position, and postoperative). ETCO2, VT, and RR (in supine and prone positions).

Preoperative parameters were recorded 24 hours preoperatively to ensure the basic results of the patient and comparing them with their changes during the study and to be sure that they are not spontaneously elevated before the study. Parameters in the supine position were recorded in the supine position after induction and before putting the patient in a prone position, while parameters in prone position were recorded after 30 minutes from putting the patient in a prone position.

As regarding VT, it was statistically significantly lower in the PCV group than the VCV group in the prone position $(\mathrm{P}=0.000)$, while there was no significant difference between the two studied groups in the supine position. As regarding ETCO2 there was no significant difference between the two studied groups in supine or prone positions. As regarding RR, it was statistically significantly lower in the PCV group than the VCV group in supine \& prone positions $(\mathrm{P}=0.004$ and 0.001 , respectively). Those were the fixed ventilation settings parameters in both PCV and VCV (Table1).

Table (1): Respiratory parameters during mechanical ventilation.

\begin{tabular}{|c|c|c|c|c|}
\hline \multicolumn{2}{|c|}{ Item } & $\begin{array}{c}\text { VCV Group } \\
(\mathbf{n}=\mathbf{3 0})\end{array}$ & $\begin{array}{c}\text { PCV Group } \\
(\mathbf{n}=\mathbf{3 0})\end{array}$ & P-value \\
\hline \multirow{2}{*}{ ETCO2 } & Supine & $34.1 \pm 1.2$ & $34.4 \pm 1.0$ & 0.238 \\
\hline & Prone & $35.0 \pm 1.1$ & $34.2 \pm 1.0$ & 0.006 \\
\hline \multirow{2}{*}{$\mathbf{R R}$} & Supine & $12.1 \pm 1.1$ & $11.3 \pm 0.99$ & $0.004 *$ \\
\hline & Prone & $10.1 \pm 0.97$ & $11.0 \pm 0.98$ & $0.001 *$ \\
\hline \multirow{2}{*}{$\mathbf{V}_{T}$} & Supine & $525.7 \pm 35.0$ & $504.0 \pm 51.1$ & 0.061 \\
\hline & Prone & $522.0 \pm 33.0$ & $443.0 \pm 39.8$ & $0.000 *$ \\
\hline \multirow{3}{*}{ PH } & Preoperative & $7.38 \pm 0.03$ & $7.38 \pm 0.02$ & 0.681 \\
\hline & Prone & $7.36 \pm 0.03$ & $7.338 \pm 0.03$ & 0.092 \\
\hline & Postoperative & $7.39 \pm 0.03$ & $7.39 \pm 0.02$ & 0.761 \\
\hline \multirow{3}{*}{ Pao2 } & Preoperative & $82.3 \pm 3.6$ & $82.1 \pm 4.03$ & 0.808 \\
\hline & Prone & $150.7 \pm 21.5$ & $166.8 \pm 17.4$ & $0.002 *$ \\
\hline & Postoperative & $82.2 \pm 3.4$ & $90.1 \pm 3.6$ & $0.000^{*}$ \\
\hline \multirow{3}{*}{ PaCo2 } & Preoperative & $38.8 \pm 3.3$ & $37.9 \pm 2.5$ & 0.226 \\
\hline & Prone & $36.1 \pm 1.9$ & $35.4 \pm 2.6$ & 0.221 \\
\hline & Postoperative & $38.4 \pm 1.9$ & $38.6 \pm 1.8$ & 0.647 \\
\hline
\end{tabular}

Regarding demographic data, there was no significant difference between the two studied groups regarding age, gender, or body mass index (BMI) (Table 2). 
Table (2): Demographic data of the studied groups.

\begin{tabular}{|c|c|c|c|c|}
\hline \multicolumn{2}{|c|}{ Item } & $\begin{array}{c}\text { VCV Group } \\
(\mathbf{n}=\mathbf{3 0})\end{array}$ & $\begin{array}{c}\text { PCV Group } \\
(\mathbf{n}=\mathbf{3 0})\end{array}$ & P-value \\
\hline \multicolumn{2}{|c|}{ Age } & $33.03 \pm 10.0$ & $31.3 \pm 9.3$ & 0.489 \\
\hline \multirow{2}{*}{ Gender } & Male & $25(83.3 \%)$ & $21(70 \%)$ & \multirow{2}{*}{0.222} \\
\hline & Female & $5(16.7 \%)$ & $9(30 \%)$ & \\
\hline \multicolumn{2}{|c|}{ BMI } & $25.4 \pm 1.9$ & $24.3 \pm 4.2$ & 0.218 \\
\hline
\end{tabular}

Regarding parameters of the systemic stress response: HR was statistically significantly lower in the PCV group than the VCV group in the prone position and postoperatively $(\mathrm{P}=0.000$ and 0.047 respectively). MAP was statistically significantly lower in the PCV group than the VCV group in the prone position and postoperatively ( $\mathrm{P}=0.000$ both). The cortisol level was statistically significantly lower in the PCV group than the VCV group in the prone position and postoperatively ( $\mathrm{P}=0.000$ both). Blood glucose level was statistically significantly lower in the PCV group than the VCV group in the prone position and postoperatively $(\mathrm{P}=0.008$ and 0.000 respectively). These results are shown in (Table 2) and (Figures 1, 2, $3,4)$.

Table (3): Perioperative arterial blood gas analysis and systemic stress response of the studied groups

\begin{tabular}{|c|c|c|c|c|}
\hline \multicolumn{2}{|c|}{ Item } & $\begin{array}{c}\text { VCV Group } \\
(\mathbf{n = 3 0})\end{array}$ & $\begin{array}{c}\text { PCV Group } \\
(\mathbf{n}=\mathbf{3 0})\end{array}$ & P-value \\
\hline \multirow{3}{*}{ HR } & Preoperative & $76.03 \pm 3.7$ & $77.8 \pm 4.8$ & 0.121 \\
\cline { 2 - 5 } & Prone & $87.2 \pm 3.9$ & $72.4 \pm 2.9$ & $0.000^{*}$ \\
\cline { 2 - 5 } & Postoperative & $74.7 \pm 2.2$ & $72.9 \pm 4.2$ & $0.047 *$ \\
\hline \multirow{3}{*}{ MAP } & Preoperative & $87.9 \pm 2.3$ & $88.0 \pm 2.5$ & 0.915 \\
\cline { 2 - 5 } & Prone & $92.5 \pm 4.3$ & $88.4 \pm 2.5$ & $0.000^{*}$ \\
\cline { 2 - 5 } & Postoperative & $81.4 \pm 2.1$ & $77.1 \pm 2.3$ & $0.000^{*}$ \\
\hline \multirow{3}{*}{ Cortisol } & Preoperative & $11.2 \pm 2.4$ & $10.7 \pm 2.6$ & 0.429 \\
\cline { 2 - 5 } & Prone & $20.7 \pm 3.8$ & $16.7 \pm 3.2$ & $0.000^{*}$ \\
\cline { 2 - 5 } & Postoperative & $21.8 \pm 4.7$ & $11.4 \pm 3.1$ & $0.000^{*}$ \\
\hline \multirow{3}{*}{ Glucose } & Preoperative & $82.1 \pm 3.3$ & $83.1 \pm 3.1$ & 0.260 \\
\cline { 2 - 5 } & Prone & $106.2 \pm 5.6$ & $99.1 \pm 13.1$ & $0.008^{*}$ \\
\cline { 2 - 5 } & Postoperative & $116.9 \pm 7.5$ & $105.5 \pm 12.1$ & $0.000^{*}$ \\
\hline
\end{tabular}




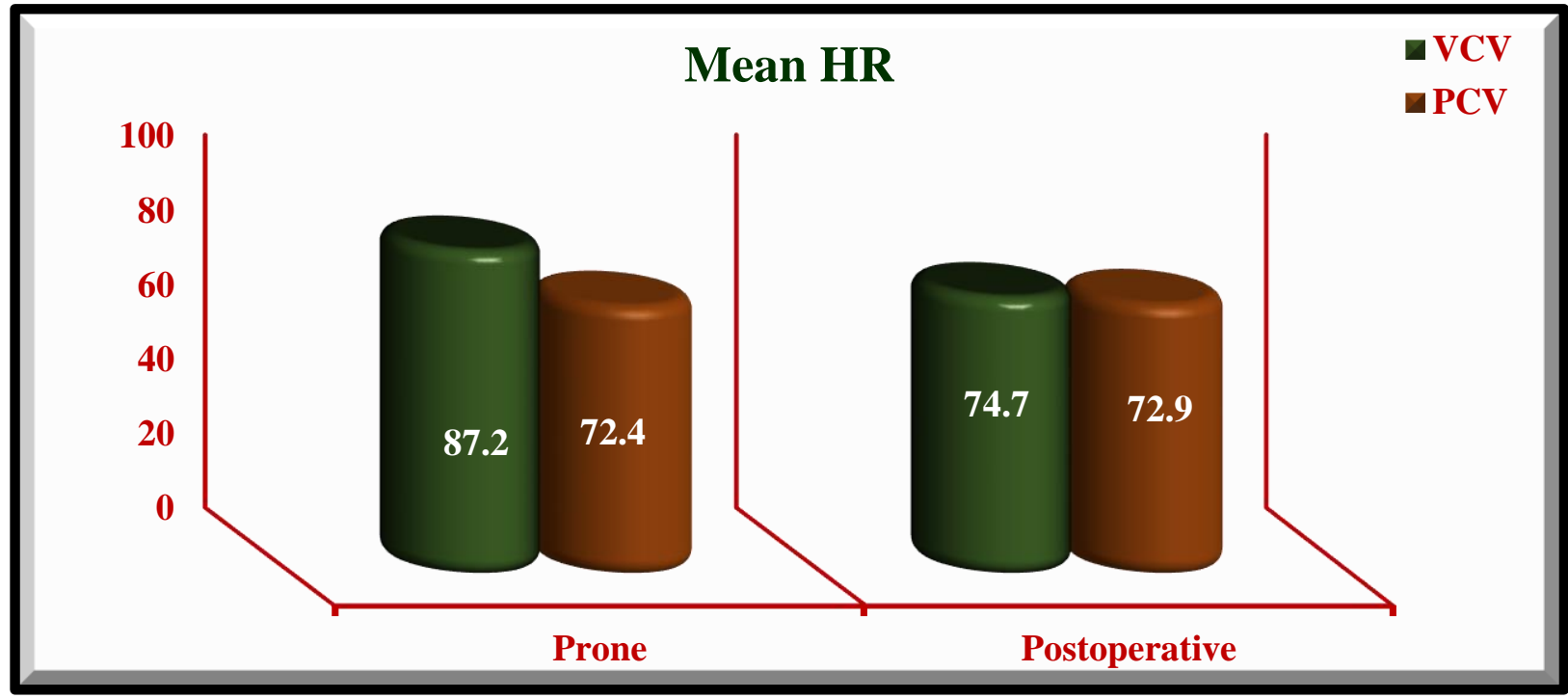

Figure (1): Comparison between the two study groups regarding HR in the prone position and postoperatively

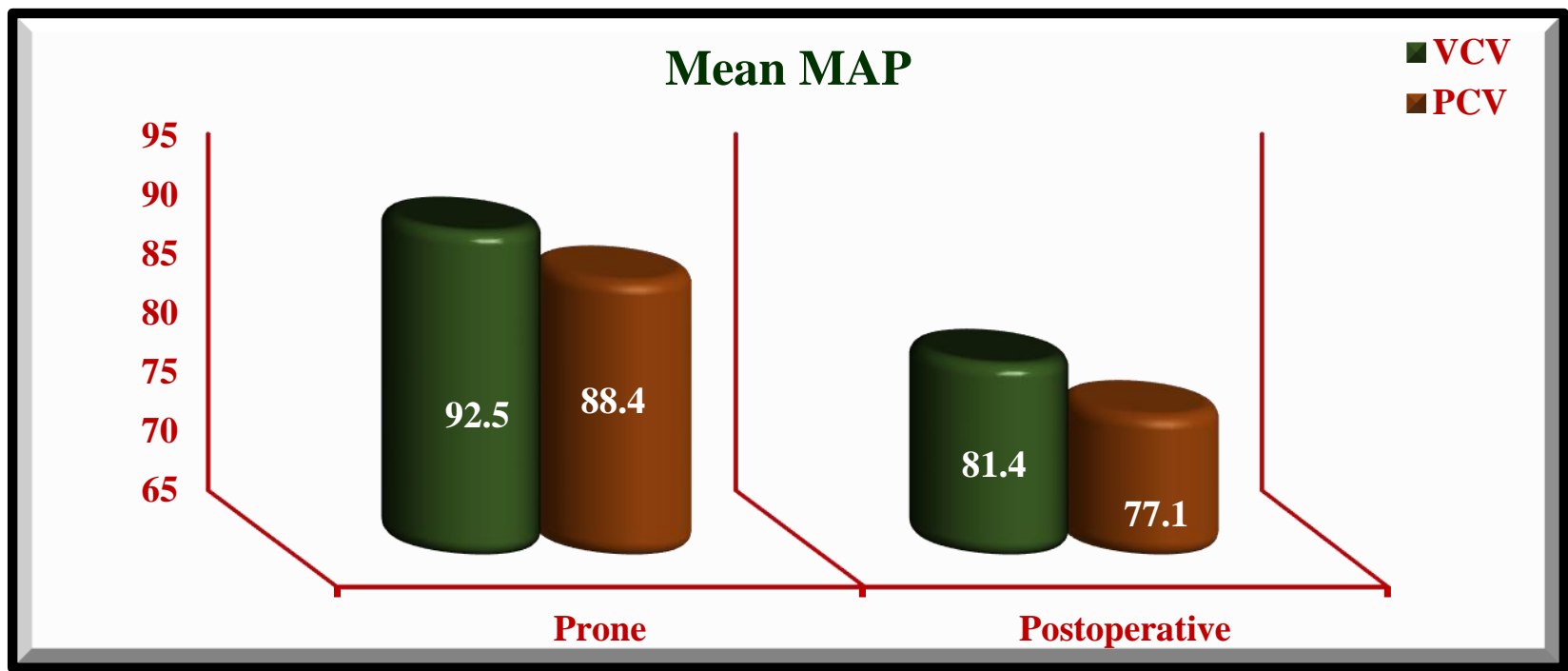

Figure (2): Comparison between the two study groups regarding MAP in the prone position and postoperatively.

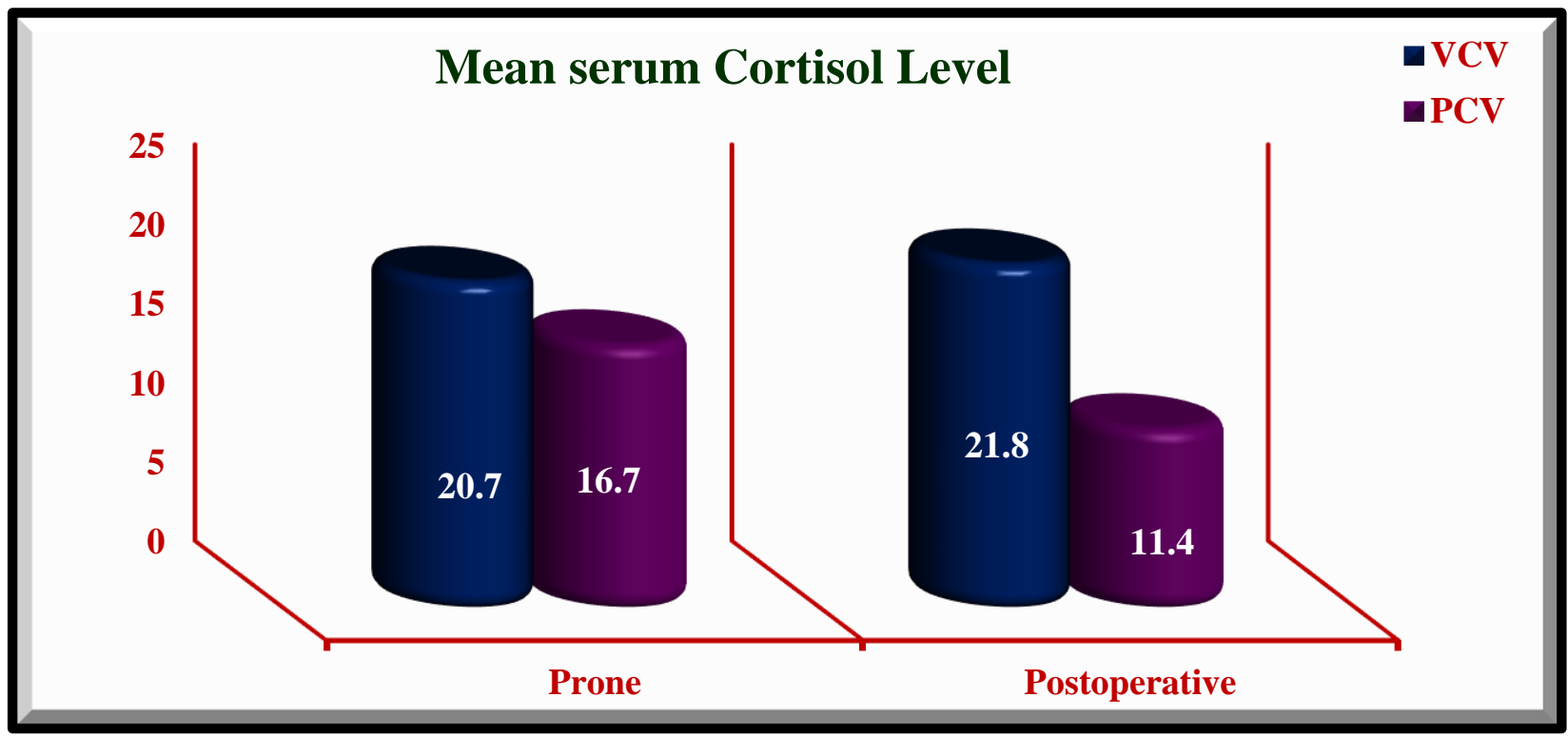

Figure (3): Comparison between the two study groups regarding serum cortisol level in the prone position and postoperatively 


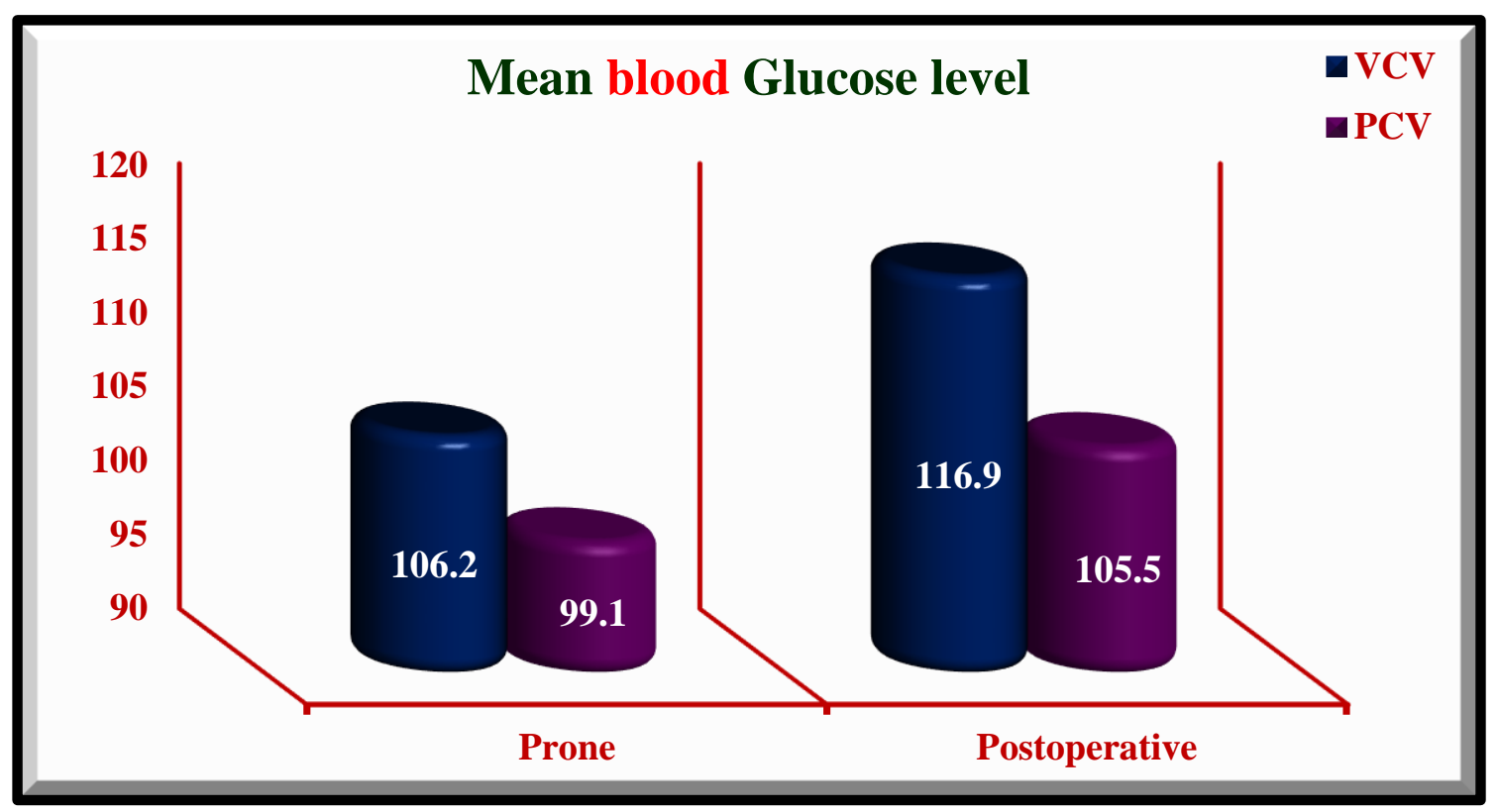

Figure (4): Comparison between the two study groups regarding serum glucose level in the prone position and postoperatively

\section{DISCUSSION}

In our study comparing the two modes of ventilation, we considered keeping the targeted VT, ETCO2 (through controlling the respiratory rate), and PEEP constant in both groups to increase the accuracy of results as these parameters are affecting airway pressure which is reflected on hemodynamic parameters. We targeted a constant VT of $(8 \mathrm{ml} / \mathrm{Kg})$ in both groups, constant ETCO2 of $(33-35 \mathrm{mmHg})$ by controlling the RR and constant PEEP of 0 (zero). Patients with a BMI $>30 \mathrm{~kg} / \mathrm{m} 2$ were excluded from this study to avoid additional confounding factors.

As regarding the constant parameters of ventilation in the two studied groups: VT was statistically significantly lower in the PCV group than the VCV group in the prone position, while there was no significant difference between the two studied groups in the supine position. Regarding ETCO2 there was no significant difference between the two studied groups in supine or prone positions and regarding RR (which used to control the ETCO2) was statistically significantly lower in the PCV group than the VCV group in supine and prone positions.

And these results confirm that these parameters were fixed during the comparison between the two modes of ventilation, in other words, fixed VT of 8 $\mathrm{ml} / \mathrm{Kg}$ and ETCO2 of 33-25 $\mathrm{mmHg}$.

Regarding stress response: Heart rate, mean arterial pressure, serum cortisol, and blood glucose levels were statistically significantly lower in the PCV group than the VCV group both intraoperatively in the prone position and postoperatively.

As we found, patients maintained on VCV showed significantly higher MAP and HR and significantly higher cortisol and blood glucose level compared to patients maintained on PCV. These findings spotlight on a fact that prone positioning and/or VCV induced increased venous engorgement resulting in increased CVP and bleeding of injured vessels during surgery. Similarly, Koh et al. ${ }^{\left(\mathbf{(}^{)}\right.}$found intraoperative blood loss was correlated with peak airway pressure changes. Malhotra et al. ${ }^{(9)}$ detected intraoperative blood loss and increased mean airway pressure at a prone position during spine surgery. Also, Kang et al. ${ }^{(10)}$ reported that PCV decreased mean arterial pressure, serum cortisol, and blood glucose in patients undergoing lumbar spine fusion surgeries. Also, Ma et al. ${ }^{(11)}$, Berger et al. ${ }^{(12)}$, and Min et al. ${ }^{(13)}$ found short-term prone positioning may have a direct effect on cardiac function with decreased stroke volume and cardiac output.

Babakhani et al. ${ }^{(7)}$ observed a significant decrease in HR and MAP accompanied by significant decreases in cerebral oxygenation at 30 and $60 \mathrm{~min}$ of prone positioning with VC. Picard et al. (14) documented that the MAP decreased below the predefined threshold in about 50\% of patients during elective spine surgery in the prone position. However, ventilation mode showed a minimal impact on $H R$ and MAP of studied patients. Hoşten et al ${ }^{\left({ }^{(15)}\right.}$ reported that the hemodynamic effects of PCV and VCV ventilation modes were found to be similar. Messeha ${ }^{(16)}$ evaluated the effect of PCV before or after VCV on hemodynamic parameters and reported no significant difference between studied patients groups. Also, Jaju et al. (17) found hemodynamic variables were comparable between patients maintained on PCV and VCV. Finally, we concluded that PCV could decrease stress response and hemodynamic effects and 
significantly improve intraoperative surgical field bleeding which may help in decreasing total blood loss during the procedure.

\section{CONCLUSION}

This study revealed that during general anaesthesia of posterior lumbar spine surgeries in the prone position it was found that comparing VCV versus PCV with keeping of the tidal volume at 8 $\mathrm{ml} / \mathrm{kg}$ and ETCO2 of $33: 35 \mathrm{mmHg}$, it was found that hemodynamic parameters including heart rate and mean arterial pressure decreased intraoperatively and postoperatively with the PCV group than with VCV group. Serum cortisol and glucose levels which are both indicators of stress response were found to be lower intraoperatively and postoperatively with the PCV group than the VCV group.

Intraoperative hemodynamic stability and decreased stress response with PCV may decrease the bleeding within the surgical field and the total blood loss. All these findings show that PCV mode has better stress response and hemodynamic stability for patients of posterior lumbar spine surgeries in the prone position.

\section{REFERENCES}

1. Davis H (1994): Increasing rates of cervical and lumbar spine surgery in the United States, 1979-1990. Spine, 19(10): 1117-23.

2. Memtsoudis S, Vougioukas V, Ma Y et al. (2011): Perioperative morbidity and mortality after anterior, posterior, and anterior/posterior spine fusion surgery. Spine, 36(22): 1867-77.

3. Pumberger M, Chiu Y, Ma Y et al. (2012): National inhospital morbidity and mortality trends after lumbar fusion surgery between 1998 and 2008. The Journal of bone and joint surgery, 94(3): 359-364.

4. Deyo R, Gray D, Kreuter W et al. (2005): United States trends in lumbar fusion surgery for degenerative conditions. Spine, 30(12): 1441-1445.

5. Pai M, Paloucek F (2000): The origin of the "ideal" body weight equations. Ann Pharmacother., 34:1066-9.

6. Rosner B (2011): Fundamentals of Biostatistics. 7th ed, vol. 8. Boston, MA: Brooks/Cole, Pp. 269-326.

7. Babakhani B, Heroabadi A, Hosseinitabatabaei $\mathrm{N}$ et al. (2017): Cerebral oxygenation under general anaesthesia can be safely preserved in patients in the prone position: A prospective observational study. J Neurosurg Anaesthesiol., 29:291-7.

8. Koh J, Lee J, Han D et al. (2013): Increase in airway pressure resulting from prone position patient placing may predict intraoperative surgical blood loss. Spine (Phila Pa), 38:678-82.

9. Malhotra A, Gupta V, Abraham M et al. (2016): Quantifying the amount of bleeding and associated changes in intra-abdominal pressure and mean airway pressure in patients undergoing lumbar fixation surgeries: A comparison of three positioning systems. Asian Spine J., 10:199-204.

10. Kang W, Oh C, Kwon W et al. (2016): Effect of mechanical ventilation mode type on intra-and postoperative blood loss in patients undergoing posterior lumbar interbody fusion surgery: A randomized controlled trial. Anaesthesiology, 125:115-23.

11. Ma M, Noori S, Maarek J et al. (2015): Prone positioning decreases cardiac output and increases systemic vascular resistance in neonates. J Perinatol., 35:424-7.

12. Berger K, Francony G, Bouzat $P$ et al. (2015): Prone position affects stroke volume variation performance in predicting fluid responsiveness in neurosurgical patients. Minerva Anaestesiol., 81:628-35.

13. Min J, Lee J, Hong $\mathbf{K}$ et al. (2017): Utility of stroke volume variation measured using non-invasive bioreactance as a predictor of fluid responsiveness in the prone position. J Clin Monit Comput., 31:397-405.

14. Picard J, Bedague $D$, Bouzat $P$ et al. (2016): Oesophageal Doppler to optimize intraoperative hemodynamics during prone position. A randomized controlled trial. Anaesth Crit Care Pain Med., 35:255-60.

15. Hoşten T, Kuş A, Gümüş E et al. (2017): Comparison of intraoperative volume and pressure-controlled ventilation modes in patients who undergo open-heart surgery. J Clin Monit Comput., 31:75-84.

16. Messeha $M$ (2017): Effect of switching between pressure -controlled and volume-controlled ventilation on respiratory mechanics and hemodynamics in obese patients during abdominoplasty. Anaesth Essays Res., 11:88-93.

17. Jaju R, Jaju P, Dubey M et al. (2017): Comparison of volume-controlled ventilation and pressure-controlled

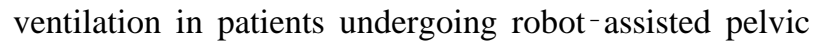
surgeries: An open-label trial. Indian J Anaesth., 61:1723. 